\title{
The Rise of PSMA Ligands for Diagnosis and Therapy of Prostate Cancer
}

\author{
Ali Afshar-Oromieh ${ }^{1,2}$, John W. Babich ${ }^{3}$, Clemens Kratochwil ${ }^{1}$, Frederik L. Giesel ${ }^{1,4}$, Michael Eisenhut ${ }^{5}$, Klaus Kopka ${ }^{4,5}$, \\ and Uwe Haberkorn ${ }^{1,2,4}$ \\ ${ }^{I}$ Department of Nuclear Medicine, Heidelberg University Hospital, Heidelberg, Germany; ${ }^{2}$ Clinical Cooperation Unit Nuclear \\ Medicine, German Cancer Research Center, Heidelberg, Germany; ${ }^{3}$ Division of Radiopharmaceutical Sciences, Department of \\ Radiology, Weill Cornell Medicine, New York, New York; ${ }^{4}$ German Cancer Consortium (DKTK), Heidelberg, Germany; and ${ }^{5}$ Division \\ of Radiopharmaceutical Chemistry, German Cancer Research Center, Heidelberg, Germany
}

\begin{abstract}
The prostate-specific membrane antigen (PSMA) has received increased consideration during the past few years as an excellent target for both imaging and therapy of prostate cancer. After many years of outstanding preclinical research, the first significant step forward in clinical use was achieved in 2008 with the first human experience with the small-molecule PSMA inhibitors ${ }^{123}$ I-MIP-1972 and ${ }^{123}$ I-MIP-1095. A clinical breakthrough followed in 2011 with ${ }^{68} \mathrm{Ga}-\mathrm{PSMA}-11$ for PET imaging and ${ }^{131}$ I-MIP-1095 for endoradiotherapy of metastatic prostate cancer. Since then, PET/CT with ${ }^{68} \mathrm{Ga}-\mathrm{PSMA}-11$ has rapidly spread worldwide, and endoradiotherapy with PSMA ligands has been conducted at increasing numbers of centers. ${ }^{68} \mathrm{Ga}-\mathrm{PSMA}-11$ is currently the subject of multicenter studies in different countries. Since 2013, ${ }^{131}$ I-related PSMA therapy has been replaced by ${ }^{177}$ Lu-labeled ligands, such as PSMA-617, which is also the subject of multicenter studies. Alternative PSMA ligands for both imaging and therapy are available. Among them is 99mTc-MIP-1404, which has recently entered a phase 3 clinical trial. This article focuses on the highlights of the development and clinical application of PSMA ligands.
\end{abstract}

Key Words: PSMA; PET/CT; prostate cancer; prostate-specific membrane antigen; endoradiotherapy

J Nucl Med 2016; 57:79S-89S

DOI: 10.2967/jnumed.115.170720

\section{$\mathbf{P}$} rostate cancer $(\mathrm{PCa})$ is the most frequent malignant tumor in men worldwide. After initial therapy, biochemical recurrence is common and is usually expressed by an elevation in prostatespecific antigen (PSA) levels. The diagnosis of recurrent $\mathrm{PCa}$ has always been challenging for conventional imaging modalities, such as CT and MRI, because of unsatisfactory sensitivity and specificity (1). So far, PET with choline derivatives has been regarded as one of the best methods for detecting recurrent PCa. However, numerous studies have reported reduced sensitivity and specificity, especially at low PSA levels and high Gleason scores

Received Mar. 31, 2016; revision accepted Jul. 19, 2016.

For correspondence contact: Ali Afshar-Oromieh, Department of Nuclear Medicine, Heidelberg University Hospital, INF 400, 69120 Heidelberg, Germany.

E-mail: a.afshar@gmx.de

COPYRIGHT (c) 2016 by the Society of Nuclear Medicine and Molecular Imaging, Inc.
(GSC) (2,3). Therefore, the development of alternative diagnostic modalities was urgently needed. In this context, prostate-specific membrane antigen (PSMA) has received increased attention during the past few years $(4,5)$.

Prostate-specific membrane antigen, also known as glutamate carboxypeptidase II, $N$-acetyl- $\alpha$-linked acidic dipeptidase I, or folate hydrolase, is a type II transmembrane glycoprotein belonging to the M28 peptidase family. The protein acts as a glutamate carboxypeptidase on various substrates, including the nutrient folate and the neuropeptide $N$-acetyl-L-aspartyl-L-glutamate (6). The PSMA protein has a unique 3-part structure: a 19-amino-acid internal portion, a 24-amino-acid transmembrane portion, and a 707amino-acid external portion (7). Prostate-specific membrane antigen is considered to be the best-established target antigen in PCa because it is highly and specifically expressed on the surface of prostate tumor cells at all tumor stages (8).

Prostate-specific membrane antigen is located in the cytosol in normal prostate cells and switches to a membrane-bound protein in prostatic carcinoma. Despite its name, PSMA is not specific for PCa. Most PSMA expression appears to be restricted to the prostate, but lower-level expression is seen in the brain, kidneys, salivary glands, and small intestine $(8,9)$. The level of PSMA expression rises with increasing tumor dedifferentiation and in metastatic and hormone-refractory cancers $(8,10,11)$. Therefore, PSMA represents an excellent target for both imaging and therapy of PCa. Since the late 1990s, it has also been well known that the neovasculature of many solid tumors may express PSMA as well (9).

\section{ANTI-PSMA MONOCLONAL ANTOBODIES}

7E11-C5.3 was the first anti-PSMA monoclonal antibody originally developed with the human PCa cell line known as LNCaP (lymph node carcinoma of the prostate) $(12,13)$. This antibody was later conjugated with diethylenetriaminepentaacetic acid, radiolabeled with ${ }^{111} \mathrm{In}$, and ultimately commercialized as an imaging agent for use with planar and SPECT imaging systems ( ${ }^{111} \mathrm{In}-$ capromab pendetide; ProstaScint; Aytu BioScience, Inc.) (14). Because capromab pendetide binds to an intracellular (cytoplasmic) epitope of PSMA, only fixed cells and necrotic cells-not intact viable cells - are "targeted" by this monoclonal antibody (13). The U.S. Food and Drug Administration approved ${ }^{111}$ In-capromab pendetide in 1996. In patients with prostate carcinoma and a high risk for metastatic disease, the sensitivity was $77 \%$ and the specificity was $86 \%$ (15). Subsequent literature reports revealed wide variations in efficacy - for example, a sensitivity of $67 \%$ for disease 
detection in the prostate bed but a sensitivity of only $10 \%$ for extraprostatic disease detection (16). This agent was a disappointment in the clinical setting, likely because of a failure to reach its target epitope on the intracellular portion of PSMA as well as poor pharmacokinetics (17).

In contrast, the monoclonal antibody J591 targets the extracellular domain of PSMA and therefore can bind viable tumor cells (18). Initially, the bifunctional chelating agent DOTA was conjugated to humanized monoclonal antibody J591 (huJ591) and labeled with ${ }^{111}$ In for imaging studies. Subsequently, huJ591 was radiolabeled with ${ }^{90} \mathrm{Y}$ and ${ }^{177} \mathrm{Lu}$ and investigated as PSMA-targeted radiotherapy for metastatic PCa. Planar and SPECT imaging studies with ${ }^{111} \mathrm{In}$ - and ${ }^{177} \mathrm{Lu}$-DOTA-huJ591 showed accurate detection of PCa metastases in bone and soft tissue as well as uptake in the tumor neovasculature of many solid tumors (19-21).

Recently, huJ591 was radiolabeled with ${ }^{89} \mathrm{Zr}$ (half-life, $78.41 \mathrm{~h}$ ) for PET imaging studies; it showed excellent tumor localization and pathologic correlation of disease $(22,23)$. A limitation of ${ }^{89} \mathrm{Zr}$ deferoxamine-J591 PET imaging is the 5- to 7-d waiting period between injection and scanning because of the long soft-tissue clearance time of the intact antibody. Localization of ${ }^{89} \mathrm{Zr}$-deferoxamineJ591 in inflammation, such as benign prostatic hypertrophy, has also been reported (22). This nonspecific uptake is not wholly unexpected because radiolabeled IgG has been used for imaging of inflammation (24).

Although radiolabeled antibodies offer promise for tumor targeting, their effectiveness as diagnostic radiopharmaceuticals is limited by a long plasma half-life, poor tumor penetrability, and nonspecific localization associated with intact immunoglobulins. Therefore, the limitation of radiolabeled antibodies is a significant delay between injection and imaging, requiring multiple visits to the imaging center by the subject. In addition, because of the long plasma half-life and time associated with normal tissue clearance, radionuclides with long half-lives are required. Hence, the radiation dosimetry of ${ }^{89} \mathrm{Zr}$ (half-life, $78.41 \mathrm{~h}$ ) is relatively high compared with that of other, short-lived positron emitters and is not favorable for repeat studies.

\section{SMALL-MOLECULE INHIBITORS OF PSMA}

Early work on the development of small-molecule inhibitors of the enzyme $\mathrm{N}$-acetylaspartylglutamate peptidase or $\mathrm{N}$-acetyl$\alpha$-linked acidic dipeptidase I, a glutamate carboxypeptidase II enzyme, identified several candidates, as described by Jackson et al. $(25,26)$. Ultimately, identification of the structural and functional

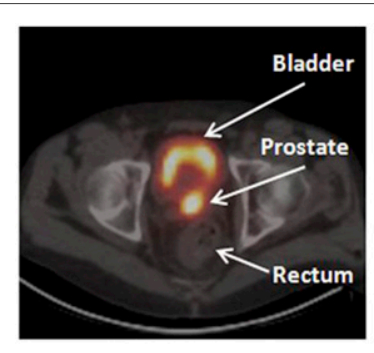

123I-MIP-1072 prostate cancer

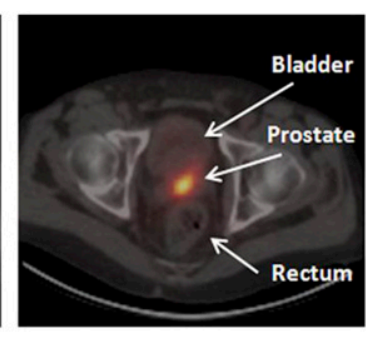

123|-MIP-1095 prostate cancer

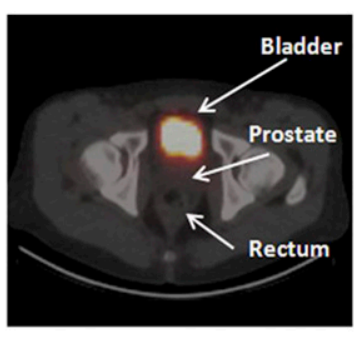

123|-MIP-1072 healthy volunteers
FIGURE 1. SPECT/CT with ${ }^{123}$ I-MIP-1072 and ${ }^{123}$ I-MIP-1095. Images are representative transaxial slices from reconstructed SPECT/CT at $4 \mathrm{~h}$ after injection of $370 \mathrm{MBq}$ of ${ }^{123} \mathrm{I}-\mathrm{MIP}-1072$ and 123/-MIP-1095. (Reprinted with permission of (33).) homology between $N$-acetyl- $\alpha$-linked acidic dipeptidase I and PSMA led to the possibility of exploiting these small molecules for the treatment and imaging of PCa via PSMA targeting $(27,28)$.

For a decade or more, efforts have been under way to develop PSMA ligands based on small-molecule inhibitors (29-36). The basic chemical structure of most PSMA ligands that have reached clinical settings incorporates glutamate-urea-glutamate or glutamateurea-lysine dimers, which are essential structural components required for binding to the catalytic domain of PSMA. These structural motifs evolved from the sequential development of the glutamate-derived hydroxyphosphinyl derivatives (e.g., phosphonomethylpentanedioic acid) described by Jackson et al. (25), the phosphinic bisdicarboxylic acid 4,4' phosphinicobis(butane-1,3dicarboxylic acid) (37), and the glutamate-urea-glutamate dimer and glutamate-urea heterodimers (29) - all originally developed with the intention to inhibit $N$-acetyl- $\alpha$-linked acidic dipeptidase I in brain tissue $(25,29)$. Thereafter, urea-based (and phosphonic acid-based) PSMA ligands were explored, developed, and continuously refined for applications in imaging and therapy of PCa (29-32,38-41).

\section{PSMA LIGAND SPECT IMAGING}

The first small-molecule inhibitors of PSMA for the imaging of PCa were introduced into the clinic in 2008 by Molecular Insight Pharmaceuticals, Inc. (MIP, now a subsidiary of Progenics Pharmaceuticals Inc.) (ClinicalTrials.gov Identifier: NCT00712829). These ligands, ${ }^{123}$ I-MIP-1072 and ${ }^{123}$ I-MIP-1095, were based on the glutamate-urea-lysine motif and contained aromatic substituents for stable introduction of the single-photon-emitting radionuclide ${ }^{123}$ I (Supplemental Fig. 1) (supplemental materials are available at http://jnm.snmjournals.org) (32,39). In men with metastatic PCa, SPECT/CT demonstrated the ability of these radioligands to rapidly detect PCa lesions in soft tissue, bone, and the prostate bed as early as $1 \mathrm{~h}$ after injection, although later images-for example, at $4 \mathrm{~h}$ after injection-demonstrated higher contrast of the lesions (Fig. 1) (33). In a subsequent evaluation of ${ }^{123}$ I-MIP-1072 in a chemotherapy model of $\mathrm{PCa}$, tumor uptake was directly proportional to viable tumor mass; this result provided initial support for the potential of PSMA imaging to track the response to therapy (41). Hence, early on, PSMA imaging appeared to have the potential to satisfy several critical unmet needs in the evaluation of PCa.

The impact of the initial human studies with these iodinated PSMA ligands was highly significant, not only because these studies demonstrated that the imaging of PCa with this approach in humans was possible but also because early results suggested that PCa could be imaged in any tissue and very soon after injection. Additionally, the use of radioiodinated ligands in the first human experience made the conceptual leap to therapy a short one; the idea was that if the pharmacokinetics and tissue distribution were favorable for either ligand, then a change in radioisotope from ${ }^{123}$ I to ${ }^{131}$ I could lead to the first targeted therapy of PCa with these small molecules. This approach was subsequently demonstrated in animals and ultimately in humans (42-44). PET studies with ${ }^{124}$ I-MIP-1095 showed high accumulation in tumor lesions, excellent contrast, and 
favorable dosimetry. The first radiotherapeutic use of smallmolecule inhibitors of PSMA in patients showed long-lasting tracer accumulation in tumor lesions and a therapeutic response with respect to PSA levels and pain symptoms in most patients.

The widespread use of ${ }^{99 \mathrm{~m}} \mathrm{Tc}$ in diagnostic nuclear medicine and of SPECT scanners in nearly all hospitals made the development of a ${ }^{99 \mathrm{~m}} \mathrm{Tc}$-based PSMA radiopharmaceutical highly desirable. To address this need, investigators at MIP developed a series of ${ }^{99 m}$ Tc-based PSMA ligands and ultimately introduced 2 high-affinity small-molecule PSMA inhibitors, MIP-1404 and MIP-1405 (Supplemental Fig. 1), into the clinical setting in 2010 $(34,45)$. Although both ${ }^{99 \mathrm{~m}} \mathrm{Tc}$ agents are based on the glutamateurea amino acid core and have similar PSMA binding affinities (34), significantly different pharmacokinetic parameters were observed in human studies; these were most likely due to modest structural differences affecting hydrophobicity and protein binding (46). In patients with metastatic PCa, localization of ${ }^{99 \mathrm{~m}} \mathrm{Tc}$ agents (MIP-1404 and MIP-1405) to lesions in bone and soft tissue correlated with radiologic evidence of metastatic disease identified by bone scanning (46). In a patient who had undergone prostatectomy and had a rising PSA level (from 1.37 to $8.9 \mathrm{ng} / \mathrm{mL}$ over a period of 4 mo), PSMA imaging with ${ }^{99 \mathrm{~m} T c-M I P-1404}$ (March) detected more metastatic lesions earlier than did the 2 bone scans obtained either before (January) or after (June) the PSMA scan (Fig. 2). These observations suggested that PSMA-targeted molecular imaging may be able to identify disease progression earlier than standard bone scanning. In addition, in several patients, significant uptake was observed in lymph nodes smaller than $10 \mathrm{~mm}$ (shortaxis diameter), considered normal by size threshold criteria used in cross-sectional imaging such as CT and MRI. On the basis of these results, a preliminary phase 1 study and a multicenter phase 2 study were conducted in high-risk PCa patients scheduled for prostatectomy and extended pelvic lymph node dissection $(47,48)$. In the phase 1 study, in all patients with GSC of greater than 7, ${ }^{99 m}$ Tc-MIP-1404 SPECT clearly identified the PCa foci in the prostate gland; the results were confirmed by histopathology and PSMA staining (47).

The findings of the phase 1 study of ${ }^{99} \mathrm{~m} T c$ agents were consistent with those of preclinical studies of PSMA-expressing LNCaP xenografts. In addition, the biodistribution, blood clearance, and tumor uptake profiles of ${ }^{99 \mathrm{~m} T c-M I P-1404}$ and ${ }^{99 \mathrm{~m}} \mathrm{Tc}-\mathrm{MIP}-1405$

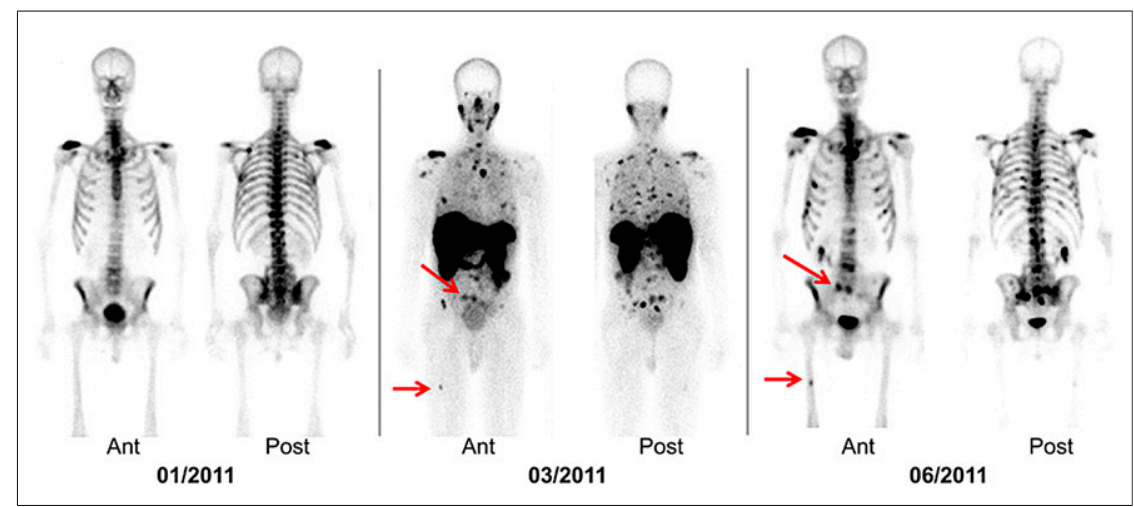

FIGURE 2. Comparison of $99 \mathrm{mT} \mathrm{T}-\mathrm{MIP}-1404$ scan with bone scans in a patient with metastatic PCa. PSMA imaging with ${ }^{99 m}$ Tc-MIP-1404 (in March) detected more metastatic lesions (arrows show two representative bone lesions) than bone scans obtained either before (in January) or after (in June) PSMA scan. Ant = anterior; Post = posterior. (Reprinted with permission of (46).) were similar to those of 2 other high-affinity PSMA inhibitors, MIP-1072 and MIP-1095, labeled with ${ }^{123} \mathrm{I}$; the latter agents were previously studied in healthy subjects and subjects with advanced metastatic cancer (33). The results of the phase 1 study demonstrated that targeting of PSMA with both ${ }^{99 \mathrm{~m} T c-M I P-1404}$ and ${ }^{99 m}$ Tc-MIP-1405 enabled the detection of radiologically proven $\mathrm{PCa}$ in bone, lymph nodes, and the prostate gland. In addition, the radiation exposure from a single administration of a diagnostic dose of either of these agents was approximately $6 \mathrm{mSv}$ - similar to the radiation exposure from a conventional bone scan in nuclear medicine. Although both of these agents showed minimal or no uptake in the prostate of healthy men, only 99mTc-MIP-1404 showed minimal urinary excretion. This finding suggested that ${ }^{99 m}$ Tc-MIP-1404 may have a distinct advantage for detecting $\mathrm{PCa}$ in the gland and in the pelvis at early stages of disease and in the case of biochemical recurrence (Supplemental Fig. 2).

The subsequent phase 2 study of ${ }^{99 m}$ Tc-MIP-1404 (trofolastat) was performed in 104 subjects who had confirmed PCa, were at high risk for extraprostatic disease (cT3 or higher or Godoy nomogram score of $\geq 130$ ), and were scheduled for a clinically indicated radical prostatectomy within $21 \mathrm{~d}$ of imaging. Patients underwent simultaneous anterior and posterior whole-body scanning followed by pelvic SPECT/CT imaging 3-6 h after injection of approximately $740 \mathrm{MBq}$ of 99mTc-MIP-1404. Semiquantitative scores of $99 \mathrm{~m}$ Tc-MIP-1404 uptake in prostate lobes and assessments of lymph node involvement were recorded by 3 nuclear medicine interpreters who were unaware of the clinical information. Quantitative measures of maximal uptake in lobes of the prostate gland versus a local background were derived from the SPECT data. All assessments were compared with pathology findings from surgical samples with GSC as the truth standard. GSC were recorded for 80 patients and ranged from $3+3$ to $5+5$. At least 2 of the 3 interpreters detected primary PCa in 98 of the 104 patients $(94 \%)$. A total of 3,025 lymph nodes were excised at surgery, and $79(2.6 \%)$ were found to be positive for PCa. The mean maximum positive lymph node size was $5.4 \mathrm{~mm}$ (range, $0.2-16 \mathrm{~mm}$ ). Interpreters identified sites of lymph node involvement in 17 of 33 patients (52\%) with pathology confirmation. The uptake of ${ }^{99 \mathrm{~m}}$ Tc-MIP-1404 in the prostate gland was significantly higher $(P<0.05)$ in patients with lymph node involvement. The results suggested that ${ }^{99 \mathrm{~m} T c-M I P-1404}$ SPECT/CT may provide prognostic information for both primary $\mathrm{PCa}$ and metastatic $\mathrm{PCa}$ in a single scan; such information may be valuable in clinical decision making (49).

On the basis of the findings of the phase 1 and phase 2 studies, a phase 3 trial with 99m Tc-MIP-1404 (trofolastat) was recently begun; ${ }^{99 \mathrm{~m} T c-M I P-1404}$ is the first smallmolecule inhibitor of PSMA to reach this developmental milestone (ClinicalTrials. gov Identifier: NCT02615067). This phase 3 study was designed to evaluate the specificity and sensitivity of 99m Tc-MIP-1404 for detecting clinically significant PCa in comparison with histopathology. In 2015, a phase 2 a study of the same tracer in men with biopsy-confirmed adenocarcinoma of the prostate gland was begun in Japan (JapicCTI-152775). ${ }^{99 \mathrm{~m} T c-M I P-1404}$ is likely the first PSMA-based radiopharmaceutical to 
achieve marketing approval for SPECT imaging in the United States. The introduction of a molecular imaging agent for PCa detection with SPECT dovetails well with recent advances in SPECT/CT technology. These advances include the introduction of digital detectors for wholebody SPECT systems; the complete integration of SPECT with CT; fast dynamic imaging capabilities; and improvements in sensitivity, resolution, and quantitation that challenge PET performance.

\section{PSMA LIGAND PET IMAGING}

The first published demonstration of PET with small-molecule inhibitors of PSMA for the imaging of PCa in animals was described by Pomper et al., who used $N$-[N-[(S)-1,3-dicarboxypropyl $]$ carbamoyl $]-$ $S$ - $\left[{ }^{11} \mathrm{C}\right]$ methyl-L-cysteine, a glutamate-urea-cysteine analog $(30)$. The first human PSMA ligand PET scan was probably conducted in October 2010 with ${ }^{18} \mathrm{~F}$-DCFBC. Recently, another ${ }^{18} \mathrm{~F}$-labeled ligand, 2-(3-\{1-carboxy-5-[(6- ${ }^{18}$ F-fluoro-pyridine-3-carbonyl)amino]-pentyl $\}$-ureido)-pentanedioic acid ( $\left.{ }^{18} \mathrm{~F}-\mathrm{DCFPyL}\right)$, was clinically introduced and showed promising results $(50,51)$.

The clinical breakthrough of PET imaging with PSMA ligands was achieved with the invention of Glu-NH-CO-NHLys-(Ahx)- ${ }^{68} \mathrm{Ga}-(\mathrm{HBED}-\mathrm{CC})\left({ }^{68} \mathrm{Ga}-\mathrm{PSMA}-11\right)$, also referred to as HBED, HBED-CC, PSMA ${ }^{\mathrm{HBED}}$, Glu-urea-Lys(Ahx)-HBED$\mathrm{CC}$, and PSMA-HBED-CC. This compound has a strong binding affinity for PSMA as well as highly efficient internalization into PCa cells $(52,53)$. The chemical formula is shown in Supplemental Figure 1. Like that for other Glu-urea-based PSMA ligands, the interactive mechanism for ${ }^{68} \mathrm{Ga}$-PSMA-11 is simple: ${ }^{68} \mathrm{Ga}$-PSMA11 binds to the extracellular part of the PSMA receptor and is then internalized into the $\mathrm{PCa}$ cell. In contrast to antibodies, ${ }^{68} \mathrm{Ga}-$ PSMA-11 is a "small molecule"- that is to say, a low-molecularweight ligand; besides its already mentioned high receptor affinity, it has excellent tissue penetration and diffuses well into solid lesions, such as osseous metastases of PCa.

Initial clinical results demonstrated that this novel method of imaging was a significant step forward in the diagnosis of recurrent PCa (54-56). In the past few years, the use of ${ }^{68} \mathrm{Ga}$-PSMA-11 $\mathrm{PET} / \mathrm{CT}$ rapidly spread worldwide.

The biodistribution of ${ }^{68} \mathrm{Ga}-\mathrm{PSMA}-11$ shows physiologic uptake in the lacrimal and salivary glands, liver, spleen, kidneys,

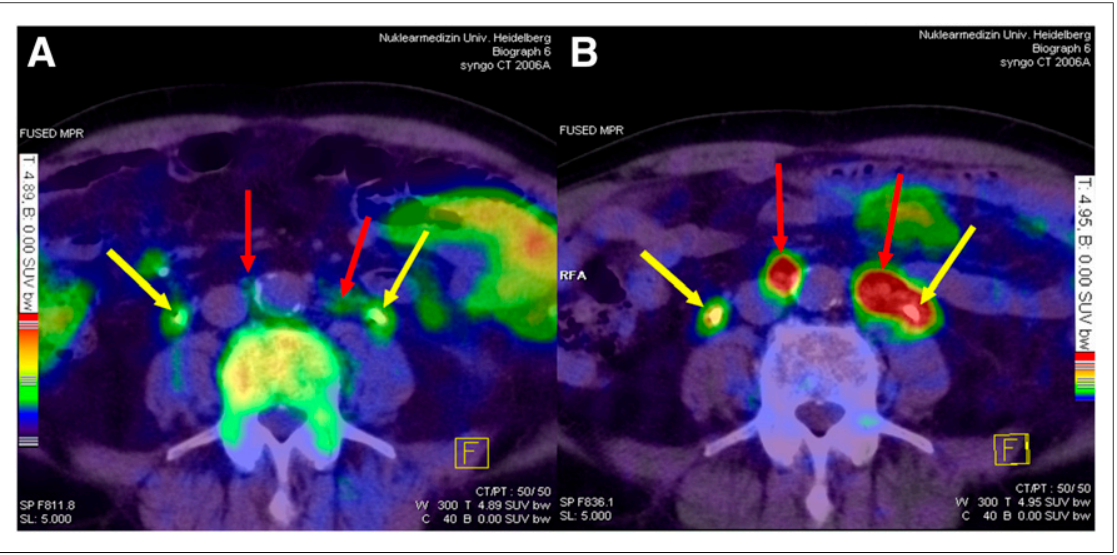

FIGURE 3. Comparison of ${ }^{18} \mathrm{~F}$-fluoromethyl-choline (A) and ${ }^{68} \mathrm{Ga}-\mathrm{PSMA}-11$ (B) in same patient. Red arrows indicate small lymph nodes that showed clearly pathologic tracer uptake on ${ }^{68} \mathrm{Ga}-\mathrm{PSMA}-11 \mathrm{PET} / \mathrm{CT}$ (B) only. Yellow arrows indicate both catheterized ureters. (A) Fusion of ${ }^{18} \mathrm{~F}$-fluoromethyl-choline PET and CT. (B) Fusion of ${ }^{68} \mathrm{Ga}$-PSMA-11 PET and CT. Color scales were automatically produced by PET/CT machine. (Reprinted with permission of (56).) and some parts of the intestines (Supplemental Fig. 3) (55,57). In addition, uptake of the compound is observed in ganglia of the vegetative nervous system, primarily with the newest generation of PET scanners (58). Unbound tracer is excreted via the kidneys and urinary tract (55).

In the first ${ }^{68} \mathrm{Ga}$-PSMA-11 PET/CT studies, tumor uptake was analyzed at 1 and $3 \mathrm{~h}$ after injection; in most PCa metastases, uptake and contrast were more intense at $3 \mathrm{~h}$ after injection than at $1 \mathrm{~h}$ after injection (55). However, because all tumor lesions already showed high contrast at $1 \mathrm{~h}$ after injection, ${ }^{68} \mathrm{Ga}$-PSMA$11 \mathrm{PET} / \mathrm{CT}$ is routinely performed at $1 \mathrm{~h}$ after injection. In addition to these data, it became evident that ${ }^{68} \mathrm{Ga}$-PSMA-11 is able to visualize even small PCa metastases with high contrast (Fig. 3; Supplemental Fig. 4).

Meanwhile, it has also been confirmed by dosimetry data that in most PCa lesions, the uptake of ${ }^{68} \mathrm{Ga}-\mathrm{PSMA}-11$ and tumor contrast increase with time: in a very small patient cohort $(n=4)$, the optimal imaging time was $3 \mathrm{~h}$ after injection (57). Alternative radiolabeled PSMA ligands, such as DCFPyL, PSMA-617, PSMA I\&T, and MIP-1095, have similar characteristics $(44,51,59,60)$. Therefore, in comparisons of different PSMA ligands, scans must be conducted within the same time frame after injection.

In another study, 37 patients with biochemical recurrence of PCa were examined with both ${ }^{18} \mathrm{~F}$-fluoromethyl-choline PET/CT and ${ }^{68} \mathrm{Ga}$-PSMA-11 PET/CT within 1 mo (56). In that comparison, ${ }^{68}$ Ga-PSMA-11 was shown to be significantly superior with regard to tracer uptake and contrast of PCa lesions, especially at low PSA levels and high GSC (Fig. 3). The SUV $\mathrm{Smax}_{\text {was }}$ at least $10 \%$ higher in approximately $79 \%$ of the detected lesions, and the contrast was higher in approximately $94 \%$ of them. A total of 78 metastases were discovered in 32 patients by ${ }^{68} \mathrm{Ga}$-PSMA PET/CT, whereas only 56 lesions were discovered in 26 patients by ${ }^{18} \mathrm{~F}$-fluoromethylcholine PET/CT. In addition, ${ }^{68}$ Ga-PSMA-11 PET/CT also had a clearly lower background signal. This feature enabled the detection of PCa lesions with low tracer uptake (Supplemental Fig. 5). Recently, these results were confirmed by another group (61).

The first evaluation with a larger patient cohort involved ${ }^{68} \mathrm{Ga}-$ PSMA-11 PET/CT of 319 patients with biochemical failure after curative-intent initial therapy (62). Overall, $82.8 \%$ of the patients had at least one lesion characteristic of $\mathrm{PCa} ; 901$ representative tumor lesions were further analyzed. The average $\mathrm{SUV}_{\text {max }}$ of these $\mathrm{PCa}$ lesions was $13.3 \pm 14.6$ (mean $\pm \mathrm{SD}$ ), with a range of 0.7-122.5.

As expected, the probability of detecting PCa lesions increased with the PSA level. At PSA levels of less than $0.2 \mathrm{ng} / \mathrm{mL}$ and between 0.2 and $0.5 \mathrm{ng} / \mathrm{mL}$, the probability of detecting tumor lesions was approximately $50 \%$. At PSA levels of between 0.5 and $1.0 \mathrm{ng} / \mathrm{mL}$, the chance of detecting tumor lesions was almost $60 \%$ and rose rapidly with PSA levels (Fig. 4). Although these detection rates seem to be low, one must remember that it is basically a great challenge for every new diagnostic method to show clear superiority over established procedures in border areas. In this context, as mentioned before, ${ }^{68} \mathrm{Ga}$-PSMA-11 PET/ CT was demonstrated to be significantly superior to ${ }^{18} \mathrm{~F}$-fluoromethyl-choline PET/CT. 
In addition, according to current knowledge, these detection rates are the highest among those of all currently known diagnostic methods for detecting recurrent PCa. Similar detection rates of ${ }^{68} \mathrm{Ga}-\mathrm{PSMA}-11 \mathrm{PET} / \mathrm{CT}$ were found by other groups $(61,63,64)$.

Various PCa types do not sufficiently express PSMA. Therefore, PSMA-based imaging may reveal no pathologic lesions despite patients' high PSA levels.

No association between faster PSA doubling times and the probability of pathologic findings on PET/CT was observed in the previously mentioned study of 319 patients. Although another study with 248 patients confirmed that observation (64), another group described such an association in a cohort of 72 patients (63). Hence, future studies with larger cohorts must address this topic in more detail.

It is known from preclinical research that PCa with higher GSC also expresses more PSMA receptors (65). In the previously mentioned analysis of 319 patients, a difference could be observed in the subgroup with very low PSA levels $(<0.2 \mathrm{ng} / \mathrm{mL})$; patients with higher GSC had a significantly higher probability of pathologic findings on ${ }^{68} \mathrm{Ga}$-PSMA-11 PET/CT (62). However, with regard to the entire patient cohort, no significant difference in tumor detection probability was found between patients with higher GSC and those with lower GSC. In contrast to that analysis of 319 patients, a separate study of 248 patients found a significant difference between patients with low GSC and those with high GSC; those with higher GSC had a significantly higher probability of pathologic findings on ${ }^{68} \mathrm{Ga}-\mathrm{PSMA}-11 \mathrm{PET} / \mathrm{CT}$ (64).

One of the most important questions about PSMA imaging concerns the role of androgen deprivation therapy (ADT). It is known from preclinical publications that ADT can increase PSMA expression in PCa cells $(66,67)$. Making such an observation in the clinical setting would have a significant impact on PSMA imaging

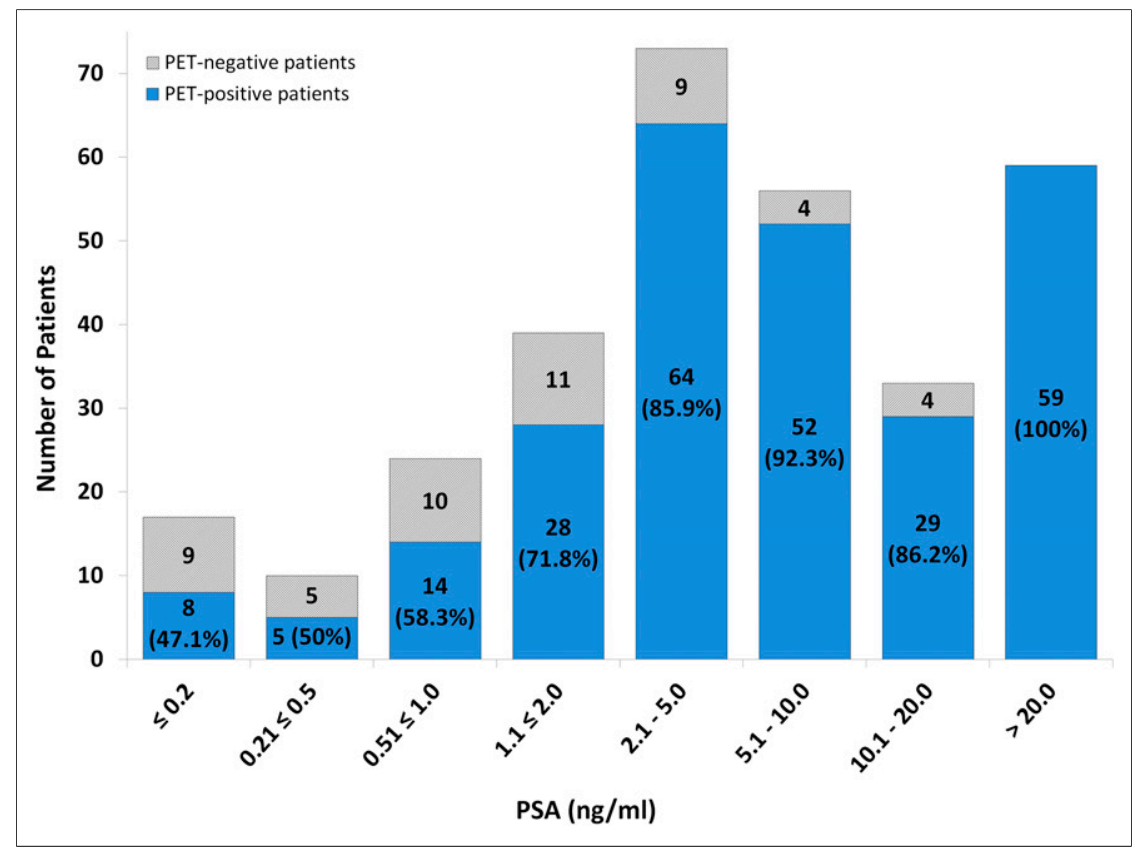

FIGURE 4. Probability of pathologic ${ }^{68} \mathrm{Ga}-\mathrm{PSMA}-11 \mathrm{PET} / \mathrm{CT}$ findings depending on PSA levels in 311 patients. Blue columns show numbers and percentages of PET/CT scans with pathologic findings. (Reprinted with permission of (62).) as well as endoradiotherapy with PSMA ligands. In the previously mentioned study of 319 patients, those who were receiving ongoing ADT had pathologic findings on ${ }^{68} \mathrm{Ga}-\mathrm{PSMA}-11 \mathrm{PET} / \mathrm{CT}$ significantly more often than those who were not, although such therapy usually leads to reductions in tumor volumes and PSA levels (62). Both of these effects usually have a negative impact on tumor detection by imaging modalities. Apart from an increase in PSMA expression in tumor lesions, another possible reason why patients with ADT had pathologic findings on ${ }^{68} \mathrm{Ga}$-PSMA-11 PET/CT significantly more often is that ADT is often started in patients with advanced-stage tumors. On the other hand, ADT is also frequently initiated in the early phases of tumor disease. Moreover, the decision to use ADT seems to be dependent on factors such as patients' preferences or urologists' experiences. In another publication, investigators could not find a significant association between ongoing $\mathrm{ADT}$ and the probability of pathologic findings on ${ }^{68} \mathrm{Ga}$-PSMA-11 PET/CT (64).

On the basis of our current knowledge and experiences, we believe that there is no indication to pause ADT before ${ }^{68} \mathrm{Ga}-$ PSMA-11 PET/CT. Nevertheless, future studies must analyze the influence of ADT on PSMA imaging and therapy in more detail.

With regard to the previously mentioned retrospective analysis of 319 patients, particular attention was paid to the available histopathologic analysis of PSMA-positive lesions (62). Specimens from 42 patients were histologically analyzed. The results for 1 local relapse in 1 patient and 29 lymph nodes in 3 other patients were false-negative. The results for all other tissues or lesions were true-positive $(n=98)$ or true-negative $(n=318)$. The lesion-based analysis revealed that sensitivity, specificity, negative predictive value, and positive predictive value were $76.6 \%, 100 \%, 91.4 \%$, and $100 \%$, respectively. The patientbased analysis revealed a sensitivity of $88.1 \%$. Calculation of specificity, negative predictive value, and positive predictive value was not possible in the patient-based analysis because virtually all patients in the cohort had recurrent disease; therefore, the cohort did not include true-negative cases. The patient-based sensitivity of ${ }^{68} \mathrm{Ga}-\mathrm{PSMA}-11 \mathrm{PET} /$ CT was calculated on the basis of this assumption; however, the validity was limited because of the lack of standardization for surgical approaches and histology. Therefore, no true gold standard for patientwise positivity or negativity existed. In particular, negative local histology findings did not imply that no tumor lesions existed in these patients.

On the basis of experiences thus far, any uptake of ${ }^{68} \mathrm{Ga}-\mathrm{PSMA}-11$ (and possibly also of alternative PSMA ligands) above the local background in lesions morphologically visible on CT or MRI is highly specific for PCa. However, this statement is currently valid only for patients with rising PSA levels after initial treatment of PCa. Recently, several case reports of different malignant and nonmalignant tumors showing uptake of ${ }^{68} \mathrm{Ga}-\mathrm{PSMA}-11$ on PET/CT were published.

The high sensitivity and excellent specificity of ${ }^{68} \mathrm{Ga}$-PSMA-11 was confirmed by other groups $(68,69)$. For example, in a recent 
study, 49 lymph nodes in 21 patients were correlated with lymph node uptake of ${ }^{68} \mathrm{Ga}$-PSMA-11 on PET/CT; all lymph nodes showed pathologic uptake, whereas $78 \%$ were morphologically inconspicuous on the CT scan $(<8 \mathrm{~mm})(70)$.

In the previously mentioned retrospective analysis of 319 patients, follow-up was done for 116 patients after ${ }^{68} \mathrm{Ga}$-PSMA$11 \mathrm{PET} / \mathrm{CT}$ (62). Of these 116 patients, 50 (43\%) were treated locally after PET/CT. Therefore, those patients could delay systemic therapy because of the results of ${ }^{68} \mathrm{Ga}$-PSMA-11 PET/CT. Patients who can delay systemic therapy have a greater potential for an improved quality of life. Systemic therapy usually has significant side effects, and the therapeutic effects are frequently only temporary. In addition, patients with locally accessible PCa theoretically have a better chance to be cured. In accordance with the results of the follow-up, another analysis demonstrated that ${ }^{68} \mathrm{Ga}$-PSMA-11 PET/CT had a dramatic impact on the application of radiotherapy and changed the management of $50.8 \%$ of the 57 patients in the cohort (71). These results confirmed findings of a previous publication (61).

${ }^{68} \mathrm{Ga}$-PSMA-11 has also been used in simultaneous PET/MRI scanners. PCa lesions are usually detected more easily and more accurately with PET/MRI than with PET/CT (Fig. 5). However, initial analyses demonstrated the frequent appearance of an extinction artifact around the urinary bladder on PET/MRI (72). This artifact, referred to as a "halo artifact," could significantly reduce the contrast and therefore the detection of PCa lesions (72). As a result, studies with PSMA ligand PET/MRI need to be interpreted with caution.

The reproducibility of hybrid PET/MRI and the ${ }^{68} \mathrm{Ga}-\mathrm{PSMA}-11$ tracer in depicting lymph node and bone metastases of PCa was compared with that of PET/CT (73). The authors observed that

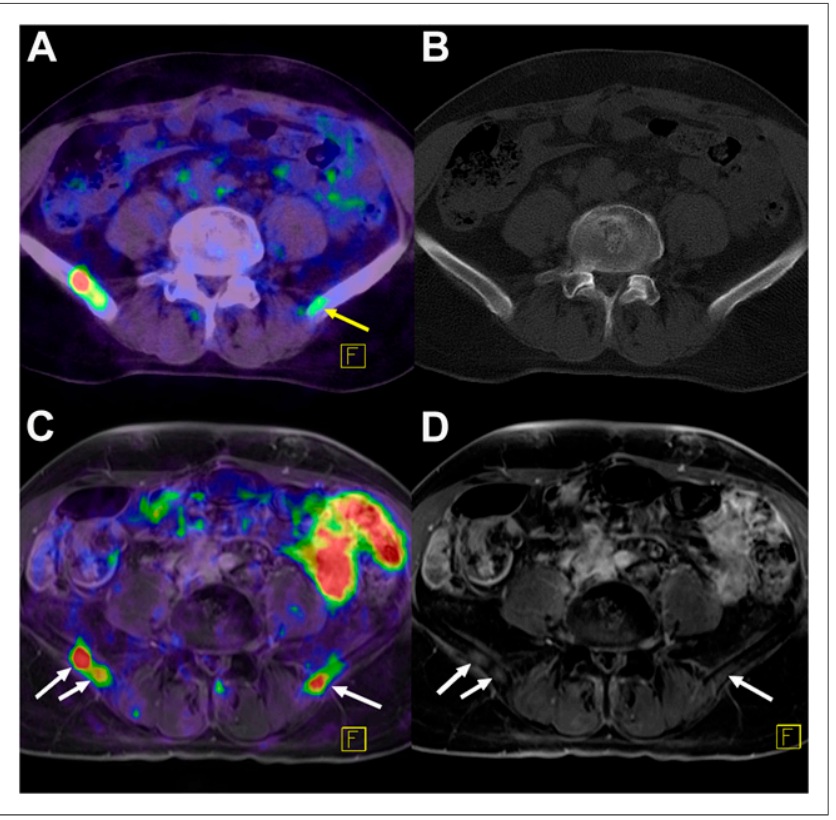

FIGURE 5. ${ }^{68} \mathrm{Ga}-\mathrm{PSMA}-11 \mathrm{PET} / \mathrm{CT}(\mathrm{A}$ and $\mathrm{B})$ and PET/MRI (C and D) of patient with recurrent $\mathrm{PCa}$. Images show potential of MRI to clarify even moderate PSMA tracer accumulations as visible on PET/CT (arrow, A); although there is no correlation on CT, arrows in D indicate pathologic MRI signals suggesting bone metastases. (A) PET/CT fusion. (B) CT without contrast medium. (C) PET/MRI fusion. (D) MRI (T1-weighted with contrast medium and fat saturation). (Reprinted with permission of (72).) nodal and osseous metastases of PCa were accurately and reliably depicted by hybrid PET/MRI with ${ }^{68} \mathrm{Ga}$-PSMA-11; discordance with PET/CT findings, including PET-positive lymph nodes of normal size, was very low (73). Another study demonstrated, for a cohort of 53 patients, that simultaneous PET/MRI with ${ }^{68} \mathrm{Ga}-$ PSMA-11 improved the diagnostic accuracy for PCa localization over that of both multiparametric MRI and PET imaging alone (74). In another recent publication, ${ }^{68} \mathrm{Ga}$-PSMA-11 PET/CT was compared with multiparametric MRI in a masked interpretation with the prostate imaging reporting and data system (PI-RADS) standard reporting schema; when MRI was considered to be a reference, the findings on ${ }^{68} \mathrm{Ga}$-PSMA-11 PET/CT were $90 \%$ concordant, whereas $97 \%$ of lesions detected by ${ }^{68} \mathrm{Ga}-\mathrm{PSMA}-11 \mathrm{PET} /$ CT had concordant findings on MRI (Supplemental Fig. 6) (75).

Despite all of this success, there is still no approval for any of the diagnostic PSMA ligands, except in Israel; in $2016,{ }^{68} \mathrm{Ga}-$ PSMA imaging was approved by the Israel Ministry of Health as a reimbursable investigation. This approval included the initial staging of patients categorized as high risk (the definition of high risk requires one of the following: localized adenocarcinoma of the prostate gland with GSC of $7-10$ or PSA levels of $>20 \mathrm{ng} / \mathrm{mL}$ or stage T3-T4) as well as biochemical failure (rise in PSA levels) after definite therapy for PCa. In all other countries, imaging methods with ${ }^{68} \mathrm{Ga}$-PSMA-11 have initially been conducted in accordance with the Helsinki Declaration and with German regulations. Initial publications about ${ }^{68} \mathrm{Ga}-\mathrm{PSMA}-11$ were used as verification for the safety and value of this PET tracer. Multicenter studies in Germany, Switzerland, Austria, and Australia are in preparation, with the aim of gaining approval of ${ }^{68} \mathrm{Ga}$-PSMA- 11 .

Table 1 shows some currently known advantages and disadvantages of PSMA imaging modalities and PSMA-targeted endoradiotherapy. However, many changes are expected in the future.

The question remains of which constellations PSMA-ligand PET/CT can be recommended for. So far, sufficient data exist for ${ }^{68} \mathrm{Ga}$-PSMA-11 only. In this context, we refer to the aforementioned probabilities of a pathologic ${ }^{68} \mathrm{Ga}$-PSMA-11 PET/CT result at different PSA values. At PSA less than $0.5 \mathrm{ng} / \mathrm{mL}$, this probability is already about 50\%. In addition, patients with higher GSC (8-10) seem to present more often with pathologic PET results. We assume that such patients can be scanned at lower PSA values than patients with GSC of 7 or lower. According to current knowledge, there is no need to pause ADT before PSMA ligand PET/CT.

The role of PSMA ligand PET imaging in the diagnosis of primary $\mathrm{PCa}$ or the primary staging of low- or high-risk PCa has not yet been sufficiently investigated. On the other hand, a phase 3 study is currently analyzing the role of PSMA ligand SPECT with ${ }^{99 \mathrm{~m}} \mathrm{Tc}-$ MIP-1404 in the diagnosis of primary PCa, as mentioned before.

\section{PSMA-TARGETED ENDORADIOTHERAPY}

As mentioned earlier, PSMA represents an excellent target for endoradiotherapy of PCa. The first ligand that was applied to patients was ${ }^{131}$ I-MIP-1095, developed by MIP. This molecule was applied to 28 patients with progressive PCa to treat metastatic PCa between 2011 and 2013 (44). These patients had already undergone all conventional treatments, and their treatment with ${ }^{131}$ I-MIP-1095 was considered to be end-of-line treatment. The patients were analyzed after receiving 1 cycle of therapy with ${ }^{131} \mathrm{I}$-MIP-1095 (mean activity, $4.8 \mathrm{GBq}$; range, 2-7.2 GBq). In a separately conducted dosimetry analysis, PCa lesions showed focal doses between 100 and 300 Gy (44). In addition, whole-body 
TABLE 1

Advantages and Disadvantages of PSMA Imaging Modalities and PSMA-Targeted Endoradiotherapy

\begin{tabular}{|c|c|c|}
\hline Technique & Advantage & Disadvantage \\
\hline \multirow[t]{4}{*}{$\begin{array}{l}\text { PSMA scintigraphy } \\
\text { (99mTc-MIP-1404) }\end{array}$} & $\begin{array}{l}\text { Primary tumor: higher } \\
\text { sensitivity than MRI }\end{array}$ & Lower spatial resolution than PET \\
\hline & Prospective trials are available & Slower pharmacokinetics than ${ }^{68} \mathrm{PSMA}-11 \mathrm{PET}$ \\
\hline & Less expensive than PET & $\begin{array}{l}\text { Value in recurrent disease has } \\
\text { not yet been analyzed }\end{array}$ \\
\hline & $\begin{array}{l}\text { Broad availability is expected in } \\
\text { future }\end{array}$ & \\
\hline \multirow[t]{2}{*}{$\begin{array}{l}\text { PSMA ligand PET/CT } \\
\left.\text { ( }{ }^{8} \mathrm{Ga}-\mathrm{PSM}-11\right)\end{array}$} & $\begin{array}{l}\text { Multiple publications are } \\
\text { available }\end{array}$ & $\begin{array}{l}\text { With one exception, only } \\
\text { retrospective analyses are available }\end{array}$ \\
\hline & $\begin{array}{l}\text { Most available PSMA imaging } \\
\text { modality worldwide }\end{array}$ & \\
\hline \multirow[t]{4}{*}{$\begin{array}{l}\text { PSMA ligand PET/MRI } \\
\text { (68 Ga-PSMA-11) }\end{array}$} & $\begin{array}{l}\text { High diagnostic value in soft } \\
\text { tissue, including primary tumor }\end{array}$ & Expensive \\
\hline & & Long scanning time \\
\hline & & $\begin{array}{l}\text { Extinction artifacts around } \\
\text { urinary bladder }\end{array}$ \\
\hline & & $\begin{array}{l}\text { Only retrospective analyses are } \\
\text { available }\end{array}$ \\
\hline \multirow[t]{3}{*}{ PSMA-targeted endoradiotherapy } & $\begin{array}{l}\text { Independent from androgen } \\
\text { receptors }\end{array}$ & $\begin{array}{l}\text { Limited data, only retrospective } \\
\text { analyses available }\end{array}$ \\
\hline & $\begin{array}{l}\text { No cross-resistance with } \\
\text { zytiga/enzalutamide/docetaxel is } \\
\text { expected }\end{array}$ & $\begin{array}{l}\text { Long-term side effects are } \\
\text { unknown }\end{array}$ \\
\hline & $\begin{array}{l}\text { Early data show good response } \\
\text { of PCa lesions }\end{array}$ & $\begin{array}{l}\text { No comparison of different } \\
\text { ligands is available }\end{array}$ \\
\hline \multirow[t]{3}{*}{ Endoradiotherapy with ${ }^{131}$ I } & Lower kidney dose & Hospitalization (5-10 d) \\
\hline & $\begin{array}{l}\text { More effective in large tumor } \\
\text { lesions than }{ }^{177} \mathrm{Lu}\end{array}$ & Bone marrow dose \\
\hline & & Radiolabeling procedure \\
\hline \multirow[t]{2}{*}{ Endoradiotherapy with ${ }^{177} \mathrm{Lu}$} & Easier radiolabeling than with ${ }^{131}$ I & Higher kidney dose than ${ }^{131}$ I \\
\hline & Short hospitalization (2 d) & \\
\hline \multirow[t]{3}{*}{ Endoradiotherapy with ${ }^{225} \mathrm{Ac}$} & $\begin{array}{l}\text { Highest therapeutic effect due to } \\
\text { a-radiation }\end{array}$ & Available at a single center worldwide \\
\hline & & Experimental \\
\hline & & Expensive \\
\hline
\end{tabular}

scintigraphic imaging demonstrated a long residence time for ${ }^{131} \mathrm{I}-\mathrm{MIP}-1095$ in PCa lesions; for example, $10 \mathrm{~d}$ after injection, PCa lesions showed uptake and contrast similar to those observed a few days after injection (44). Within the first 3 mo after the administration of ${ }^{131} \mathrm{I}$-MIP-1095, PSA levels decreased by at least $50 \%$ in $60.7 \%$ of the patients and by at least $75 \%$ in $25 \%$ of the patients. PSMA imaging frequently showed a significant reduction in tumor masses after endoradiotherapy (Fig. 6). Fourteen percent of the patients were nonresponders. The average time until PSA progression was $126 \mathrm{~d}$ (range, 62-469 d). In this patient cohort, no correlation was found between the applied amounts of activity and a decrease in PSA levels or the time to PSA progression after a decrease in PSA levels.

Because of the high physiologic uptake of PSMA ligands in salivary glands, side effects were expected in these organs. Seven of the 28 patients experienced transient xerostomia. In one case, mucositis was reported. However, all reported symptoms resolved within 3-4 wk. With regard to blood counts, mainly (transient) thrombocytopenia was reported. Leukopenia was registered rarely and presented with lymphocytopenia.

Since 2013, ${ }^{131}$ I-related PSMA therapy has been replaced by therapy with ${ }^{177} \mathrm{Lu}$ - and ${ }^{225} \mathrm{Ac}$-labeled therapeutic ligands, such as PSMA-617 (Supplemental Fig. 1). ${ }^{225}$ Ac-labeled PSMA-targeted therapy is currently available in Heidelberg, Germany, only. Since 2013, ${ }^{177} \mathrm{Lu}-\mathrm{PSMA}-617$ has been increasingly used for radioligand therapy of metastatic $\mathrm{PCa}$ patients in different countries and is the subject of multicenter studies in Germany and Australia (ACTRN12615000912583).

One of the aims of these studies is the approval of PSMA- 617. As with diagnostic ligands, there is currently no approval for 


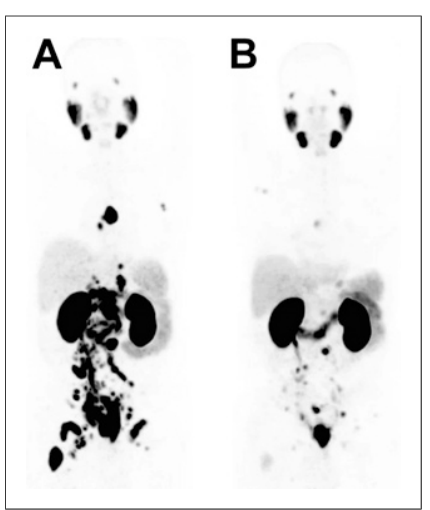

FIGURE 6. ${ }^{68} \mathrm{Ga}-\mathrm{PSMA}-11$ PET/ CT of patient before $(A)$ and 3 mo after (B) 1 cycle of ${ }^{131}$ I-MIP-1095. (Reprinted with permission of (44).) therapeutic PSMA ligands. Therefore, the application is allowed only for "compassionate use" in cases of progressive $\mathrm{PCa}$ after all other approved therapeutic modalities have been applied. PSMA-617 consists of the Glu-urea motif and the chelator DOTA separated by a lipophilic linker region; it has the characteristics of PSMA-11 and further refinements in pharmacokinetics, particularly reduced kidney uptake. In preclinical studies, PSMA-617 showed significantly improved binding affinity as well as highly efficient internalization into LNCaP cells, and tumor-to-background ratios of up to 1,058 were observed at $24 \mathrm{~h}$ after injection (76). Because of the DOTA chelator, this ligand can be labeled with, for example, ${ }^{68} \mathrm{Ga},{ }^{44} \mathrm{Sc},{ }^{111} \mathrm{In},{ }^{90} \mathrm{Y},{ }^{177} \mathrm{Lu},{ }^{213} \mathrm{Bi}$, and ${ }^{225} \mathrm{Ac}$. Therefore, it can be used for both imaging and therapy.

${ }^{68}$ Ga-PSMA-617 has already been successfully used in PET/CT imaging (59). Nevertheless, the high-level performance of ${ }^{68} \mathrm{Ga}$-PSMA-617 in the diagnosis of $\mathrm{PCa}$ is offset by slightly slower tracer kinetics than for PSMA-11, which might be caused by the larger size of PSMA-617 than of PSMA-11; therefore, only images at $3 \mathrm{~h}$ after injection could benefit from the improved internalization rate (59).

The favorable dosimetry of ${ }^{177} \mathrm{Lu}$-PSMA-617 has been confirmed by centers in Istanbul, Munich, Cologne, and Heidelberg (77-81). In brief, the red marrow dose is approximately $0.03 \mathrm{~Gy} / \mathrm{GBq}$, and the kidney dose is $0.5-0.7 \mathrm{~Gy} / \mathrm{GBq}$; the salivary glands and lacrimal glands are the dose-limiting organs, with doses of 1.2-2.8 Gy/GBq. Tumor doses of 1.2-47.5 Gy/GBq (mean, 13.1 Gy/GBq) have been calculated.

One of the earliest patients treated with ${ }^{177} \mathrm{Lu}-\mathrm{PSMA}-617$ received 2 cycles of ${ }^{177}$ Lu-PSMA-617 in February 2014 and May

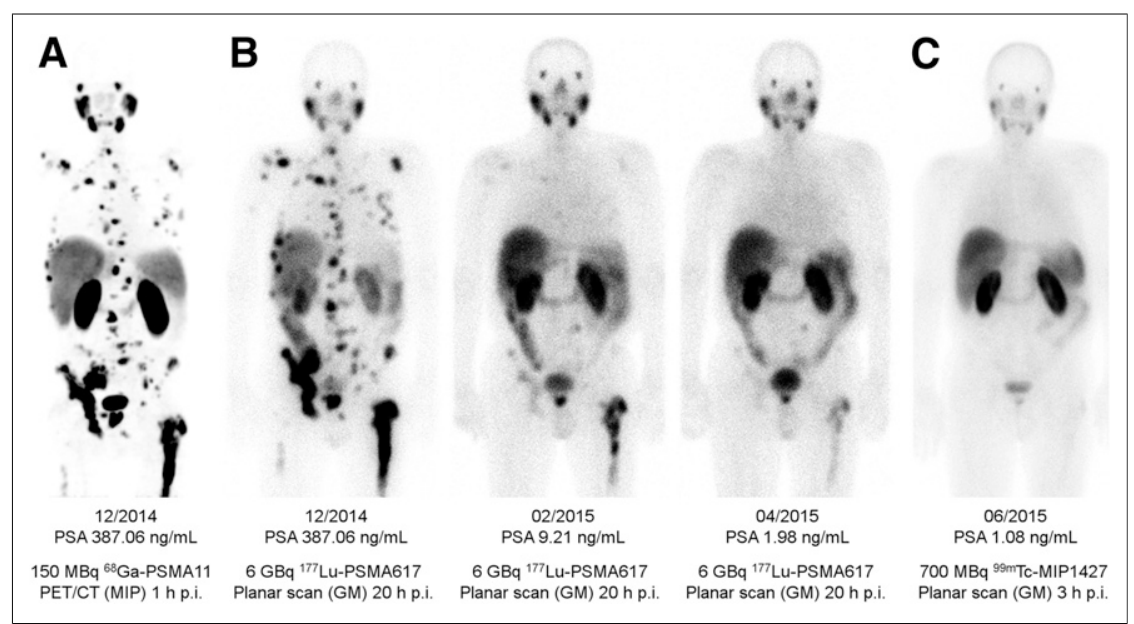

FIGURE 7. Different imaging modalities for 1 patient. (A) PSMA ligand PET/CT delivers highest resolution. (B) Coemission of $y$-rays enables imaging during therapy. (C) 99mTc-PSMA scintigraphy has minimally less noise than posttherapy scans and can be used for imaging follow-up in outpatient setting. GM = geometric mean; MIP = maximum-intensity projection; p.i. = after injection. (Reprinted with permission of (81).)
2014, with a cumulative activity of $7.4 \mathrm{GBq}$, and showed a radiologically complete response (80). After his case was published and presented at several congresses, the interest in PSMA-targeted radionuclide therapy increased dramatically.

Since 2013, ${ }^{177} \mathrm{Lu}-\mathrm{PSMA}-617$ has been increasingly used for radioligand therapy of metastatic PCa patients in multiple centers. The current experience is based only on "compassionate use" of the compound outside prospective clinical trials. Nevertheless, in recent years, there have been several case reports of patients with a marked response to PSMA radioligand therapy, even in challenging situations $(82,83)$. More structured data have been reported recently. In a case series of 24 patients receiving 2 cycles of ${ }^{177} \mathrm{Lu}-\mathrm{PSMA}-617$, with a mean dose of $6 \mathrm{GBq} /$ cycle, the PSA response rate after the first cycle was approximately $80 \%$. However, some patients had an early relapse, and in only $70 \%$ of the patients was the PSA response sustained until 8-10 wk after the second cycle (84). Another publication reported 30 patients receiving up to 3 cycles of ${ }^{177} \mathrm{Lu}-$ PSMA-617 therapy bimonthly, with a typical treatment activity of $6 \mathrm{GBq}(81)$. A PSA response of $70 \%$ after cycle 1 was found (Fig. 7). Again, the response was of limited duration in a portion of the patients, and only about $50 \%$ of the patients had an ongoing response until the restaging was performed at week 24 -that is, 6 mo and 3 cycles after the initiation of treatment. Beside a few patients who died early after therapy because of tumor progression, both centers reported only grade 1 or 2 hematologic toxicities and sporadically mild xerostomia and fatigue as side effects. Another group administered ${ }^{177} \mathrm{Lu}-\mathrm{DOTAGA}-(\mathrm{I}-\mathrm{y}) \mathrm{fk}(\mathrm{Sub}-\mathrm{KuE})$ to a cohort of 56 patients $(85)$.

Although results are very preliminary and were obtained from heterogeneous groups of patients, the PSA response rate, progressionfree survival, and good tolerance of the treatment are highly encouraging for the further evaluation of PSMA-targeted radionuclide therapy in prospective clinical trials.

\section{CONCLUSION}

In December 2015, the PSMA ligand SPECT tracer ${ }^{99 m}$ Tc-MIP1404 entered phase 3 clinical trials in men with biopsy-proven lowgrade PCa in the United States and Canada. In 2015, the same tracer entered into a phase $2 \mathrm{a}$ study in men with biopsy-confirmed presence of adenocarcinoma of the prostate gland in Japan. ${ }^{99 \mathrm{~m}} \mathrm{Tc}-\mathrm{MIP}-1404$ likely will be the first PSMA-based radiopharmaceutical to achieve marketing approval for SPECT imaging in the United States. The introduction of a molecular imaging agent for PCa detection by SPECT dovetails well with recent advances in SPECT/CT technology.

PET/CT with ${ }^{68} \mathrm{Ga}$-PSMA-11 is routinely performed at $1 \mathrm{~h}$ after injection. However, later imaging shows most lesions of PCa with higher contrast and uptake and therefore can help to clarify equivocal lesions. ${ }^{68}$ Ga-PSMA-11 PET/CT proved to be significantly superior to choline PET/CT, primarily at low PSA levels and high GSC.

In the largest patient cohort analyzed so far $(n=319), 82.8 \%$ of patients who had a biochemical relapse of $\mathrm{PCa}$ showed at least one lesion characteristic of $\mathrm{PCa}$ on ${ }^{68} \mathrm{Ga}-$ PSMA-11 PET/CT. The probability of 
pathologic ${ }^{68} \mathrm{Ga}$-PSMA-11 PET/CT findings was dependent on PSA levels. At PSA levels of less than $0.5 \mathrm{ng} / \mathrm{mL}$, the probability of detecting PCa lesions was approximately $50 \%$ and rose rapidly at higher PSA levels. The impact of GSC on PET imaging with PSMA needs to be analyzed further. However, higher GSC seem to be associated with higher rates of pathologic PET findings. Patients receiving ADT at the time of ${ }^{68} \mathrm{Ga}-\mathrm{PSMA}-11 \mathrm{PET} / \mathrm{CT}$ more frequently had positive PET scan results than patients not receiving such treatment. According to a lesion-based analysis, sensitivity, specificity, negative predictive value, and positive predictive value were $76.6 \%, 100 \%, 91.4 \%$, and $100 \%$, respectively. Of all patients available for follow-up, $40 \%$ received local therapy after ${ }^{68} \mathrm{Ga}-\mathrm{PSMA}-11 \mathrm{PET} / \mathrm{CT}$ and therefore could delay systemic therapy for $\mathrm{PCa}$.

The PSMA receptor is also well suited for endoradiotherapy. The first experience of 1 cycle of PSMA therapy with ${ }^{131}$ I-MIP1095 in 28 patients between 2011 and 2013 showed promising results; in $60.7 \%$ of the patients, PSA levels dropped by at least $50 \%$. Dosimetry analysis revealed values of up to $300 \mathrm{~Gy}$ in $\mathrm{PCa}$ lesions. Since 2013, ${ }^{177}$ Lu-PSMA-617 has been increasingly used for radioligand therapy of metastatic PCa patients in several centers worldwide. The favorable dosimetry of PSMA-617 has been confirmed by different centers. Several publications reported PSA response rates of $70 \%-80 \%$ in patients receiving up to 3 cycles of ${ }^{177} \mathrm{Lu}-\mathrm{PSMA}-617$. In a portion of patients, the response was of limited duration (a few weeks), and only about $50 \%$ of the patients had an ongoing response until restaging was performed. Only grade 1 or 2 hematologic toxicities and sporadically mild xerostomia and fatigue were reported as side effects. However, long-term side effects have not yet been evaluated. Although results are very preliminary and were obtained from heterogeneous groups of patients, the PSA response rate, progression-free survival, and good tolerance of the treatment are highly encouraging for the further evaluation of PSMA-targeted radionuclide therapy in prospective clinical trials.

\section{DISCLOSURE}

No potential conflict of interest relevant to this article was reported.

\section{REFERENCES}

1. Baek SE, Lee KH, Park YS, et al. RNA aptamer-conjugated liposome as an efficient anticancer drug delivery vehicle targeting cancer cells in vivo. J Control Release. 2014;196:234-242.

2. Schmid DT, John H, Zweifel R, et al. Fluorocholine PET/CT in patients with prostate cancer: initial experience. Radiology. 2005;235:623-628.

3. Igerc I, Kohlfurst S, Gallowitsch HJ, et al. The value of ${ }^{18} \mathrm{~F}$-choline PET/ CT in patients with elevated PSA-level and negative prostate needle biopsy for localisation of prostate cancer. Eur J Nucl Med Mol Imaging. 2008;35: 976-983.

4. Maffioli L, Florimonte L, Costa DC, et al. New radiopharmaceutical agents for the treatment of castration-resistant prostate cancer. Q J Nucl Med Mol Imaging. 2015;59:420-438.

5. Santoni M, Scarpelli M, Mazzucchelli R, et al. Targeting prostate-specific membrane antigen for personalized therapies in prostate cancer: morphologic and molecular backgrounds and future promises. J Biol Regul Homeost Agents. 2014;28:555-563.

6. Carter RE, Feldman AR, Coyle JT. Prostate-specific membrane antigen is a hydrolase with substrate and pharmacologic characteristics of a neuropeptidase. Proc Natl Acad Sci USA. 1996;93:749-753.

7. Grauer LS, Lawler KD, Marignac JL, Kumar A, Goel AS, Wolfert RL. Identification, purification, and subcellular localization of prostate-specific membrane antigen $\mathrm{PSM}^{\prime}$ protein in the $\mathrm{LNCaP}$ prostatic carcinoma cell line. Cancer Res. 1998;58:4787-4789.

8. Israeli RS, Powell CT, Corr JG, Fair WR, Heston WD. Expression of the prostate-specific membrane antigen. Cancer Res. 1994;54:1807-1811.

9. Chang SS. Overview of prostate-specific membrane antigen. Rev Urol. 2004; 6(suppl 10):S13-S18.

10. Wright GL Jr, Grob BM, Haley C, et al. Upregulation of prostate-specific membrane antigen after androgen-deprivation therapy. Urology. 1996;48: $326-334$.

11. Sweat SD, Pacelli A, Murphy GP, Bostwick DG. Prostate-specific membrane antigen expression is greatest in prostate adenocarcinoma and lymph node metastases. Urology. 1998;52:637-640.

12. Horoszewicz JS, Kawinski E, Murphy GP. Monoclonal antibodies to a new antigenic marker in epithelial prostatic cells and serum of prostatic cancer patients. Anticancer Res. 1987;7:927-935.

13. Troyer JK, Beckett ML, Wright GL. Location of prostate-specific membrane antigen in the LNCaP prostate carcinoma cell line. Prostate. 1997; 30:232-242.

14. Rosenthal SA, Haseman MK, Polascik TJ. Utility of capromab pendetide (ProstaScint) imaging in the management of prostate cancer. Tech Urol. 2001;7:27-37.

15. Hinkle GH, Burgers JK, Neal CE, et al. Multicenter radioimmunoscintigraphic evaluation of patients with prostate carcinoma using indium-111 capromab pendetide. Cancer. 1998;83:739-747.

16. Schuster DM, Taleghani PA, Nieh PT, et al. Characterization of primary prostate carcinoma by anti-1-amino-2-[ $\left.{ }^{18} \mathrm{~F}\right]$-fluorocyclobutane-1-carboxylic acid (anti-3[ $\left.\left.{ }^{18} \mathrm{~F}\right]-\mathrm{FACBC}\right)$ uptake. Am J Nucl Med Mol Imaging. 2013;3:85-96.

17. Wilkinson $\mathrm{S}$, Chodak $\mathrm{G}$. The role of ${ }^{111}$ indium-capromab pendetide imaging for assessing biochemical failure after radical prostatectomy. J Urol. 2004; 172:133-136.

18. Vallabhajosula S, Smith-Jones PM, Navarro V, Goldsmith SJ, Bander NH. Radioimmunotherapy of prostate cancer in human xenografts using monoclonal antibodies specific to prostate specific membrane antigen (PSMA): studies in nude mice. Prostate. 2004;58:145-155.

19. Vallabhajosula S, Kuji I, Hamacher KA, et al. Pharmacokinetics and biodistribution of ${ }^{111} \mathrm{In}$ - and ${ }^{177} \mathrm{Lu}$-labeled J591 antibody specific for prostate-specific membrane antigen: prediction of ${ }^{90} \mathrm{Y}-\mathrm{J} 591$ radiation dosimetry based on ${ }^{111} \mathrm{In}$ or ${ }^{177}$ Lu? J Nucl Med. 2005;46:634-641.

20. Bander NH, Milowsky MI, Nanus DM, Kostakoglu L, Vallabhajosula S, Goldsmith SJ. Phase I trial of ${ }^{177}$ lutetium-labeled J591, a monoclonal antibody to prostate-specific membrane antigen, in patients with androgen-independent prostate cancer. J Clin Oncol. 2005;23:4591-4601.

21. Tagawa ST, Akhtar NH, Christos PJ, et al. Noninvasive measurement of prostatespecific membrane antigen (PSMA) expression with radiolabeled J591 imaging: a prognostic tool for metastatic castration-resistant prostate cancer (CRPC) [abstract]. J Clin Oncol. 2013;31(suppl):11081.

22. Osborne JR, Green DA, Spratt DE, et al. A prospective pilot study of ${ }^{89} \mathrm{Zr}-\mathrm{J} 591 /$ prostate specific membrane antigen positron emission tomography in men with localized prostate cancer undergoing radical prostatectomy. J Urol. 2014;191: 1439-1445.

23. Morris MJ, Pandit-Taskar N, Tagawa ST, et al. Tumor-directed PET imaging of bone metastases in metastatic castration-resistant prostate cancer (mCRPC) using Zr-89 labeled anti-prostate specific membrane antigen (PSMA) antibody J591 [abstract]. J Clin Oncol. 2014;32(suppl 4):25.

24. De Gersem R, Jamar F. Nonspecific human immunoglobulin G for imaging infection and inflammation: what did we learn? Q J Nucl Med Mol Imaging. 2010;54:617-628.

25. Jackson PF, Cole DC, Slusher BS, et al. Design, synthesis, and biological activity of a potent inhibitor of the neuropeptidase $\mathrm{N}$-acetylated alpha-linked acidic dipeptidase. J Med Chem. 1996;39:619-622.

26. Jackson PF, Slusher BS. Design of NAALADase inhibitors: a novel neuroprotective strategy. Curr Med Chem. 2001;8:949-957.

27. Luthi-Carter R, Barczak AK, Speno H, Coyle JT. Molecular characterization of human brain $N$-acetylated alpha-linked acidic dipeptidase (NAALADase). J Pharmacol Exp Ther. 1998;286:1020-1025.

28. Tiffany CW, Lapidus RG, Merion A, Calvin DC, Slusher BS. Characterization of the enzymatic activity of PSM: comparison with brain NAALADase. Prostate. 1999;39:28-35.

29. Kozikowski AP, Nan F, Conti P, et al. Design of remarkably simple, yet potent urea-based inhibitors of glutamate carboxypeptidase II (NAALADase). J Med Chem. 2001;44:298-301.

30. Pomper MG, Musachio JL, Zhang J, et al. ${ }^{11} \mathrm{C}-\mathrm{MCG}$ : synthesis, uptake selectivity, and primate PET of a probe for glutamate carboxypeptidase II (NAALADase). Mol Imaging. 2002;1:96-101. 
31. Kozikowski AP, Zhang J, Nan F, et al. Synthesis of urea-based inhibitors as active site probes of glutamate carboxypeptidase II: efficacy as analgesic agents. J Med Chem. 2004;47:1729-1738.

32. Maresca KP, Hillier SM, Femia FJ, et al. A series of halogenated heterodimeric inhibitors of prostate specific membrane antigen (PSMA) as radiolabeled probes for targeting prostate cancer. J Med Chem. 2009;52:347-357.

33. Barrett JA, Coleman RE, Goldsmith SJ, et al. First-in-man evaluation of 2 highaffinity PSMA-avid small molecules for imaging prostate cancer. J Nucl Med. 2013;54:380-387.

34. Hillier SM, Maresca KP, Lu G, et al. ${ }^{99 \mathrm{~m}} \mathrm{Tc}$-labeled small-molecule inhibitors of prostate-specific membrane antigen for molecular imaging of prostate cancer. J Nucl Med. 2013;54:1369-1376.

35. Banerjee SR, Pullambhatla M, Foss CA, et al. ${ }^{64} \mathrm{Cu}$-labeled inhibitors of prostatespecific membrane antigen for PET imaging of prostate cancer. J Med Chem. 2014;57:2657-2669.

36. Eder M, Eisenhut M, Babich J, Haberkorn U. PSMA as a target for radiolabelled small molecules. Eur J Nucl Med Mol Imaging. 2013;40:819-823.

37. Nan F, Bzdega T, Pshenichkin S, et al. Dual function glutamate-related ligands: discovery of a novel, potent inhibitor of glutamate carboxypeptidase II possessing mGluR3 agonist activity. J Med Chem. 2000;43:772-774.

38. Foss CA, Mease RC, Fan H, et al. Radiolabeled small-molecule ligands for prostate-specific membrane antigen: in vivo imaging in experimental models of prostate cancer. Clin Cancer Res. 2005;11:4022-4028.

39. Hillier SM, Maresca KP, Femia FJ, et al. Preclinical evaluation of novel glutamate-urea-lysine analogues that target prostate-specific membrane antigen as molecular imaging pharmaceuticals for prostate cancer. Cancer Res. 2009;69:6932-6940.

40. Banerjee SR, Pullambhatla M, Byun Y, et al. ${ }^{68} \mathrm{Ga}$-labeled inhibitors of prostatespecific membrane antigen (PSMA) for imaging prostate cancer. J Med Chem. 2010;53:5333-5341.

41. Hillier SM, Kern AM, Maresca KP, et al. ${ }^{123}$ I-MIP-1072, a small-molecule inhibitor of prostate-specific membrane antigen, is effective at monitoring tumor response to taxane therapy. J Nucl Med. 2011;52:1087-1093.

42. Hillier S, Rubino K, Maresca K, et al. [ $\left.{ }^{131} \mathrm{I}\right] \mathrm{MIP}-1466$, a small molecule prostatespecific membrane antigen (PSMA) inhibitor for targeted radiotherapy of prostate cancer (PCa) [abstract]. Soc Nucl Med Annu Meet Abstr. 2012;53:170.

43. Hillier S, Merkin R, Maresca K, et al. [ ${ }^{131}$ I]MIP-1375, a small molecule prostatespecific membrane antigen (PSMA) inhibitor for targeted therapy of prostate cancer (PCa) [abstract]. J Nucl Med. 2011;52(suppl 1):361.

44. Zechmann CM, Afshar-Oromieh A, Armor T, et al. Radiation dosimetry and first therapy results with a ${ }^{124} \mathrm{I} /{ }^{131} \mathrm{I}$-labeled small molecule (MIP-1095) targeting PSMA for prostate cancer therapy. Eur J Nucl Med Mol Imaging. 2014;41: 1280-1292.

45. Lu G, Maresca KP, Hillier SM, et al. Synthesis and SAR of ${ }^{99 \mathrm{~m}} \mathrm{Tc} / \mathrm{Re}-$ labeled small molecule prostate specific membrane antigen inhibitors with novel polar chelates. Bioorg Med Chem Lett. 2013;23:1557-1563.

46. Vallabhajosula S, Nikolopoulou A, Babich JW, et al. ${ }^{99 \mathrm{~m}} \mathrm{Tc}-$ labeled smallmolecule inhibitors of prostate-specific membrane antigen: pharmacokinetics and biodistribution studies in healthy subjects and patients with metastatic prostate cancer. J Nucl Med. 2014;55:1791-1798.

47. Vallabhajosula S, Osborne J, Nikolopoulou A, et al. PSMA targeted SPECT imaging biomarker to detect local and metastatic prostate cancer (PCa): phase I studies with ${ }^{99 m}$ Tc-MIP-1404 [abstract]. Soc Nucl Med Annu Meet Abstr. 2013;54:281.

48. Goffin K, Joniau S, Tenke P, et al. A phase 2 study of ${ }^{99 \mathrm{~m}} \mathrm{Tc}$-trofolastat (MIP1404) to identify prostate cancer (PCa) in high-risk patients (pts) undergoing radical prostatectomy (RP) and extended pelvic lymph node (ePLN) dissection: an interim analysis [abstract]. Soc Nucl Med Annu Meet Abstr. 2014; $55: 15$.

49. Dabasi G, Barra M, Tenke P, et al. Correlation of technetium Tc99m trofolastat chloride (MIP-1404) uptake using SPECT/CT with histopathology: a phase 2 study of prostate cancer (PCa) patients undergoing radical prostatectomy (RP) with extended lymph node dissection (ePLND). Eur J Nucl Med Mol Imaging. 2014;41(suppl):S236-S237.

50. Chen Y, Pullambhatla M, Foss CA, et al. 2-(3-\{1-Carboxy-5-[(6- $\left[{ }^{18} \mathrm{~F}\right]$ fluoropyridine-3-carbonyl)-amino]-pentyl $\}$-ureido)-pentanedioic acid, $\left[{ }^{18} \mathrm{~F}\right] \mathrm{DCFPyL}$, a PSMA-based PET imaging agent for prostate cancer. Clin Cancer Res. 2011; 17:7645-7653.

51. Szabo Z, Mena E, Rowe SP, et al. Initial evaluation of $\left[{ }^{18} \mathrm{~F}\right] \mathrm{DCFPyL}$ for prostatespecific membrane antigen (PSMA)-targeted PET imaging of prostate cancer. Mol Imaging Biol. 2015;17:565-574.

52. Eder M, Schäfer M, Bauder-Wüst U, et al. ${ }^{68}$ Ga-complex lipophilicity and the targeting property of a urea-based PSMA inhibitor for PET imaging. Bioconjug Chem. 2012;23:688-697.
53. Eder M, Neels O, Müller M, et al. Novel preclinical and radiopharmaceutical aspects of $\left[{ }^{68} \mathrm{Ga}\right] \mathrm{Ga}-\mathrm{PSMA}-\mathrm{HBED}-\mathrm{CC}$ : a new PET tracer for imaging of prostate cancer. Pharmaceuticals (Basel). 2014;7:779-796.

54. Afshar-Oromieh A, Haberkorn U, Eder M, Eisenhut M, Zechmann CM. $\left[{ }^{68} \mathrm{Ga}\right]$ gallium-labelled PSMA ligand as superior PET tracer for the diagnosis of prostate cancer: comparison with ${ }^{18} \mathrm{~F}-\mathrm{FECH}$. Eur J Nucl Med Mol Imaging. 2012;39:1085-1086.

55. Afshar-Oromieh A, Malcher A, Eder M, et al. PET imaging with a $\left[{ }^{68} \mathrm{Ga}\right]$ galliumlabelled PSMA ligand for the diagnosis of prostate cancer: biodistribution in humans and first evaluation of tumour lesions. Eur J Nucl Med Mol Imaging. 2013;40:486-495.

56. Afshar-Oromieh A, Zechmann CM, Malcher A, et al. Comparison of PET imaging with a ${ }^{68} \mathrm{Ga}$-labelled PSMA ligand and ${ }^{18} \mathrm{~F}$-choline-based PET/CT for the diagnosis of recurrent prostate cancer. Eur J Nucl Med Mol Imaging. 2014;41: 11-20.

57. Afshar-Oromieh A, Hetzheim H, Kübler W, et al. Radiation dosimetry of ${ }^{68} \mathrm{Ga}-$ PSMA-11 (HBED-CC) and preliminary evaluation of optimal imaging timing. Eur J Nucl Med Mol Imaging. 2016;43:1611-1620.

58. Krohn T, Verburg FA, Pufe T, et al. $\left[{ }^{68} \mathrm{Ga}\right]$ PSMA-HBED uptake mimicking lymph node metastasis in coeliac ganglia: an important pitfall in clinical practice. Eur J Nucl Med Mol Imaging. 2015;42:210-214.

59. Afshar-Oromieh A, Hetzheim H, Kratochwil C, et al. The theranostic PSMA ligand PSMA-617 in the diagnosis of prostate cancer by PET/CT: biodistribution in humans, radiation dosimetry, and first evaluation of tumor lesions. J Nucl Med. 2015;56:1697-1705.

60. Herrmann K, Bluemel C, Weineisen M, et al. Biodistribution and radiation dosimetry for a probe targeting prostate-specific membrane antigen for imaging and therapy. J Nucl Med. 2015;56:855-861.

61. Morigi JJ, Stricker PD, van Leeuwen PJ, et al. Prospective comparison of ${ }^{18} \mathrm{~F}-$ fluoromethylcholine versus ${ }^{68} \mathrm{Ga}$-PSMA PET/CT in prostate cancer patients who have rising PSA after curative treatment and are being considered for targeted therapy. J Nucl Med. 2015;56:1185-1190.

62. Afshar-Oromieh A, Avtzi E, Giesel FL, et al. The diagnostic value of PET/ CT imaging with the ${ }^{68} \mathrm{Ga}$-labelled PSMA ligand HBED-CC in the diagnosis of recurrent prostate cancer. Eur J Nucl Med Mol Imaging. 2015;42: 197-209.

63. Ceci F, Uprimny C, Nilica B, et al. ${ }^{68}$ Ga-PSMA PET/CT for restaging recurrent prostate cancer: which factors are associated with PET/CT detection rate? Eur J Nucl Med Mol Imaging. 2015;42:1284-1294.

64. Eiber M, Maurer T, Souvatzoglou M, et al. Evaluation of hybrid ${ }^{68}$ Ga-PSMA ligand PET/CT in 248 patients with biochemical recurrence after radical prostatectomy. J Nucl Med. 2015;56:668-674.

65. Marchal C, Redondo M, Padilla M, et al. Expression of prostate specific membrane antigen (PSMA) in prostatic adenocarcinoma and prostatic intraepithelial neoplasia. Histol Histopathol. 2004;19:715-718.

66. Evans MJ, Smith-Jones PM, Wongvipat J, et al. Noninvasive measurement of androgen receptor signaling with a positron-emitting radiopharmaceutical that targets prostate-specific membrane antigen. Proc Natl Acad Sci USA. 2011; 108:9578-9582.

67. Liu T, Wu LY, Fulton MD, Johnson JM, Berkman CE. Prolonged androgen deprivation leads to downregulation of androgen receptor and prostatespecific membrane antigen in prostate cancer cells. Int J Oncol. 2012;41: 2087-2092.

68. Herlemann A, Wenter V, Kretschmer A, et al. ${ }^{68} \mathrm{Ga}$-PSMA positron emission tomography/computed tomography provides accurate staging of lymph node regions prior to lymph node dissection in patients with prostate cancer. Eur Urol. January 19, 2016 [Epub ahead of print].

69. Maurer T, Gschwend JE, Rauscher I, et al. Diagnostic efficacy of ${ }^{68}$ galliumPSMA positron emission tomography compared to conventional imaging for lymph node staging of 130 consecutive patients with intermediate to high risk prostate cancer. J Urol. 2016;195:1436-1443.

70. Giesel FL, Fiedler H, Stefanova M, et al. PSMA PET/CT with Glu-urea-Lys-(Ahx)$\left[{ }^{68} \mathrm{Ga}\right.$ (HBED-CC)] versus 3D CT volumetric lymph node assessment in recurrent prostate cancer. Eur J Nucl Med Mol Imaging. 2015;42:1794-1800.

71. Sterzing F, Kratochwil C, Fiedler H, et al. ${ }^{68}$ Ga-PSMA-11 PET/CT: a new technique with high potential for the radiotherapeutic management of prostate cancer patients. Eur J Nucl Med Mol Imaging. 2016;43:34-41.

72. Afshar-Oromieh A, Haberkorn U, Schlemmer HP, et al. Comparison of PET/CT and PET/MRI hybrid systems using a ${ }^{68}$ Ga-labelled PSMA ligand for the diagnosis of recurrent prostate cancer: initial experience. Eur J Nucl Med Mol Imaging. 2014;41:887-897.

73. Freitag MT, Radtke JP, Hadaschik BA, et al. Comparison of hybrid ${ }^{68} \mathrm{Ga}-$ PSMA PET/MRI and ${ }^{68} \mathrm{Ga}$-PSMA PET/CT in the evaluation of lymph node 
and bone metastases of prostate cancer. Eur J Nucl Med Mol Imaging. 2016;43:70-83.

74. Eiber M, Weirich G, Holzapfel K, et al. Simultaneous ${ }^{68}$ Ga-PSMA HBED-CC PET/MRI improves the localization of primary prostate cancer. Eur Urol. January 18, 2016 [Epub ahead of print].

75. Giesel FL, Sterzing F, Schlemmer HP, et al. Intra-individual comparison of ${ }^{68} \mathrm{Ga}$-PSMA-11-PET/CT and multi-parametric MR for imaging of primary prostate cancer. Eur J Nucl Med Mol Imaging. 2016;43:1400-1406.

76. Benešová $M$, Schäfer $M$, Bauder-Wüst $U$, et al. Preclinical evaluation of a tailor-made DOTA-conjugated PSMA inhibitor with optimized linker moiety for imaging and endoradiotherapy of prostate cancer. J Nucl Med. 2015;56: 914-920.

77. Kabasakal L, AbuQbeitah M, Aygün A, et al. Pre-therapeutic dosimetry of normal organs and tissues of ${ }^{177}$ Lu-PSMA-617 prostate-specific membrane antigen (PSMA) inhibitor in patients with castration-resistant prostate cancer. Eur J Nucl Med Mol Imaging. 2015;42:1976-1983.

78. Delker A, Fendler WP, Kratochwil C, et al. Dosimetry for ${ }^{177}$ Lu-DKFZ-PSMA617: a new radiopharmaceutical for the treatment of metastatic prostate cancer. Eur J Nucl Med Mol Imaging. 2016;43:42-51.
79. Hohberg M, Eschner W, Schmidt M, et al. Lacrimal glands may represent organs at risk for radionuclide therapy of prostate cancer with $\left[{ }^{177} \mathrm{Lu}\right]$ DKFZ-PSMA617. Mol Imaging Biol. 2016;18:437-445.

80. Kratochwil C, Giesel FL, Eder M, et al. $\left[{ }^{177} \mathrm{Lu}\right]$ lutetium-labelled PSMA ligandinduced remission in a patient with metastatic prostate cancer. Eur J Nucl Med Mol Imaging. 2015;42:987-988.

81. Kratochwil C, Giesel FL, Stefanova M, et al. PSMA-targeted radionuclide therapy of metastatic castration-resistant prostate cancer with ${ }^{177} \mathrm{Lu}$-labeled PSMA617. J Nucl Med. 2016;57:1170-1176.

82. Soydal C, Ozkan E, Akyurek S, Kucuk NO. Marked response to ${ }^{177} \mathrm{Lu}$ prostatespecific membrane antigen treatment in patient with metastatic prostate cancer. Clin Nucl Med. 2016;41:159-160.

83. Schlenkhoff CD, Gaertner F, Essler M, Schmidt M, Ahmadzadehfar H. Positive influence of ${ }^{177} \mathrm{Lu}$ PSMA-617 therapy on bone marrow depression caused by metastatic prostate cancer. Clin Nucl Med. 2016;41:478-480.

84. Ahmadzadehfar H, Eppard E, Kürpig S, et al. Therapeutic response and side effects of repeated radioligand therapy with ${ }^{177} \mathrm{Lu}$-PSMA-DKFZ-617 of castrateresistant metastatic prostate cancer. Oncotarget. 2016;7:12477-12488.

85. Baum RP, Kulkarni HR, Schuchardt C, et al. ${ }^{177}$ Lu-labeled prostate-specific membrane antigen radioligand therapy of metastatic castration-resistant prostate cancer: safety and efficacy. J Nucl Med. 2016;57:1006-1013. 